\title{
Strain dependent grain growth during cold rolling deformation of gradient nanograined nickel
}

\author{
Haitao $\mathrm{Ni}^{1,2}$, Jiang $\mathrm{Zhu}^{1}$, Heshan Yang ${ }^{1, *}$, Yong $\mathrm{Pu}^{3}$, Lixia Wang ${ }^{4}$ \\ ${ }^{1}$ College of Chemistry and Environmental Engineering, Chongqing University of Arts and Sciences, Yongchuan 402160, China. \\ ${ }^{2}$ Chongqing Key Laboratory of Environmental Materials \& Remediation Technologies, Chongqing University of Arts and Sciences, \\ Yongchuan 402160, China. \\ ${ }^{3}$ College of Materials Science and Engineering, Chongqing University of Arts and Sciences, Yongchuan 402160, China. \\ ${ }^{4}$ College of Intelligent Manufacturing Engineering, Chongqing University of Arts and Sciences, Yongchuan 402160, China.
}

\begin{abstract}
The effect of the rolling strain on grain growth behavior of two gradient nanograined nickel samples (average grain size ranging from 20 90nm) with symmetrical structure was investigated by scanning electron microscope/transmission electron microscopy observation, X-ray line profile analysis and microhardness measurement. In both gradient microstructures, under the same volume fraction, the layer with small grains and the layer with large grains was systematically compared. Quantitative analysis indicated that at a given nominal rolling strain small grains seem to grow more slowly than large grains, which can be attributed to the fact that the "hard" small grains sustain less deformation when the gradient are deformed to a certain strain.
\end{abstract}

\section{Introduction}

Gradient nanostructured materials have become a research focus of the materials and mechanics community because of their excellent combination of strength and plasticity. Recently a series of investigations on the gradient nanostructured metals have revealed some understandings on the relationship between the overall performance and the gradient structure have been obtained [1-3].

Throughout the tensile deformation behavior of gradient nanostructured metals, there are some great differences in the evolution of microstructure and performance when compared to their homogeneous counterparts [4-5]. From the results of published research on the rolling deformation of homogeneous nanograined metals and alloys, the deformation mechanism is closely related to the grain size. For the electrodeposited homogeneous nanograined nickel, it is generally accepted that larger grains typically tend to deform via full dislocations, whereas smaller grains typically tend to deform via partial dislocations [6-7]. In light of this, our previous work on the rolling deformation of grain-size gradient nanograined nickel has preliminarily reported microstructure evolution and the corresponding microhardness variation of several typical layers with different grain size levels [8]. However, the volume fractions of the typical layers in the two samples are different. In this study, to better examine the grain growth behavior of the deformed gradient sample, the composing proportion of gradient microstructure is almost the same by sample preparation.

\section{Materials and Methods}

Two groups of gradient nanograined nickel samples with symmetrical structure were prepared by electrodeposition following the procedure described in the previous literature [9], except for electrodeposition time at given current density varying $1.5 \sim 15 \mathrm{~A} / \mathrm{dm} 2$. Rolling deformation was conducted to achieve similar nominal rolling strain reported in our previous publication [8].

The microstructure observations of the samples were carried out by scanning electron microscope (SEM) and transmission electron microscope (TEM) studies. SEM observation was conducted by Carl Zeiss GeminiSEM300 operated at $10 \mathrm{kV}$ using the InLens mode. TEM observation was conducted by FEI TECNAI 20 operated at $200 \mathrm{kV}$.

XRD measurement was performed on a Rigaku D/MAX 2500 PC diffractometer with a rotating copper target $(18 \mathrm{KW})$. The diffraction data were obtained in a fixed time mode (a step time of $0.5 \mathrm{~s}$ ) from $40^{\circ}$ to $100^{\circ}$ using a step of $0.02^{\circ}$. Quantitative information concerning microstructural parameters was determined by X-ray line profile analysis.

Due to the limited macroscopic size of the sample, the mechanical response was characterized by microhardness measurement, using a load of $0.98 \mathrm{~N}$ and a dwell time of 10s.

*yheshan@cqwu.edu.cn 


\section{Results and discussion}

Figure 1 shows the schematic diagram of the overall grain size distribution along the thickness direction in two groups of gradient samples. It can be seen that the microstructure gradient is composed of similar grain zone with similar thickness, but in opposite sequence. For simplicity, we hereafter refer to the samples with large grains in the surface zone as the sample A, while the samples with small grains in the surface zone as the sample B. Particularly, typical SEM images of the large grain zone and small grain zone are also displayed. Further statistical results on grain size distribution indicate that the sizes of small grains range from $10 \mathrm{~nm}$ to $50 \mathrm{~nm}$ whereas the sizes of large grains range from $50 \mathrm{~nm}$ to $140 \mathrm{~nm}$.
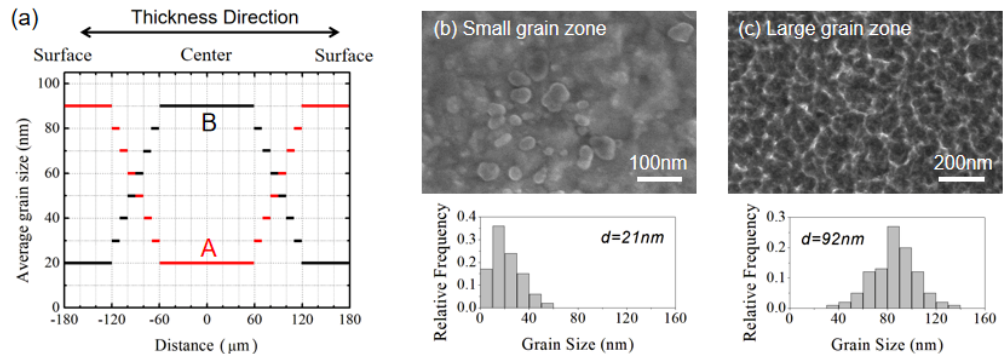

Figure 1 Overall grain size distribution in two groups of gradient samples

Figure 2 shows typical bright-field TEM images of the surface and center layers in the two samples after final deformation. It is not strange that an obvious grain coarsening is observed. Even during the deformation of gradient microstructure, the nanograins with relative high surface free energy inevitably have a strong tendency to destabilize under the applied external stress [10]. Considering that different microstructure evolution behaviors caused by different strain component, the grain size distribution in the surface and center layers are investigated by conventional grain size statistics. From the size distribution results, it can be found that the size variation of larger grains seems to be relative higher than

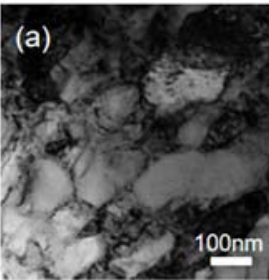

(e)

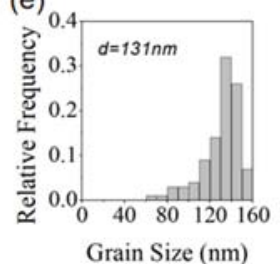

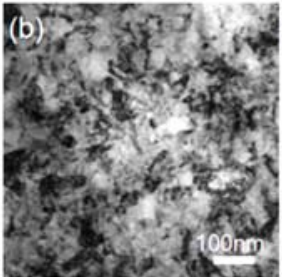

(f)

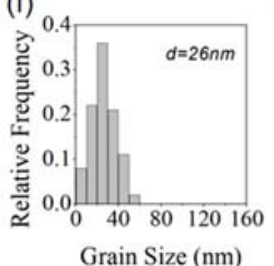

that of small grains, no matter where in the sample they locate. In addition, for the grains with similar size range, the size variation of the grains in the surface layer seem to be relative higher than that of the grains in the center layers, which is in agreement with our previous studies. Nevertheless, the visible difference in the average grain size deserves to be noted. There should be some differences between grains with different size levels, because the volume fractions of various grain zones with different sizes are distinctly different from the previous design of gradient microstructures.

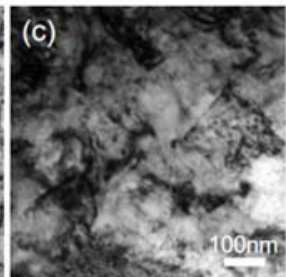

(g)

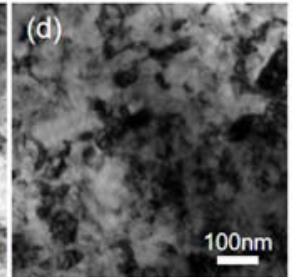

(h)
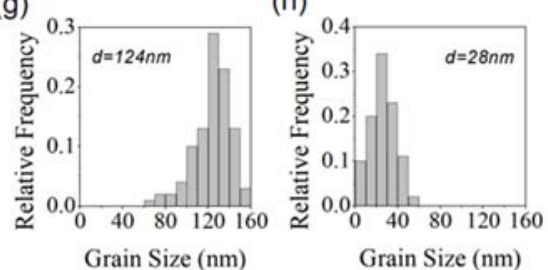

Figure 2 Typical bright-field TEM images and corresponding grain size distributions of the final deformed samples: (a) and (e) the surface layer of the sample A; (b) and (f) the center layer of the sample A; (c) and (g) the center layer of the sample B; (d) and (h) the surface layer of the sample B.

To better understand the effect of strain gradient on the grain growth behavior, average grain sizes at different nominal rolling strains after each deformation were determined by x-ray diffraction line profile analysis. The grain size evolution overall the deformation process is shown in Figure 3. Although the grain sizes determined by XRD analysis are lower than those determined by SEM/TEM measurement, mainly owing to the fact that the XRD results mostly reflect the size of the sub-grains, the evolution of grain size provides a clear evidence for the difference in grain size distribution determined by microscopic observation. Comparing with the previous results on another gradient structure, it is noteworthy that the size increase of small grain is relatively small and the size increase of large grain is relatively large in this study. According to the deformation mechanism of gradient structure metals [11], it is probably because the layers with small grains, as the "hard" phase in the gradient microstructure, sustain less deformation, while the "soft" layers with large grains should sustain more deformation when the sample was deformed to a given nominal rolling strain. 

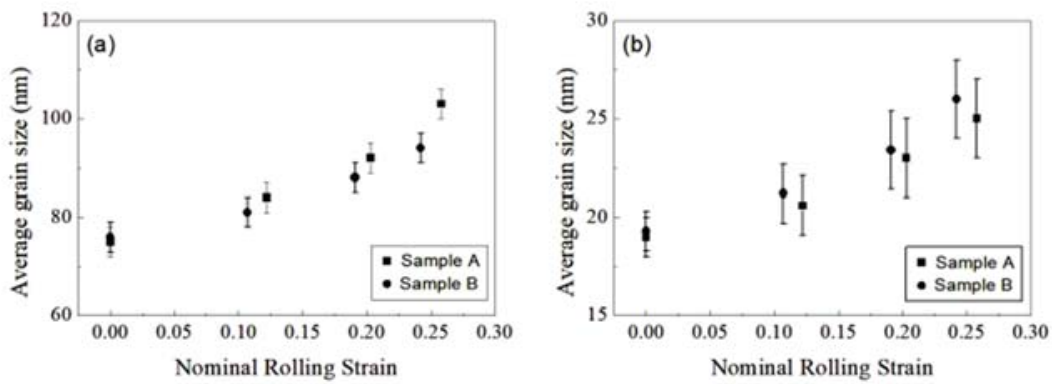

Figure 3 Size evolution of the typical layers with (a) large grains and (b) small grains overall the rolling deformation at various nominal rolling strains

Figure 4 shows microhardness evolution during overall deformation. Despite the fact that the grains are coarsen to a certain extent by deformation, an obvious microhardness increase, the so-called strain hardening, is still found in the surface and center layers. However, the increment of microhardness for the layers with small

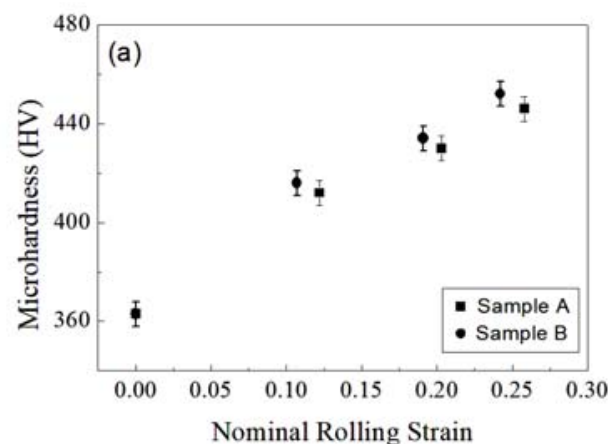

Figure 4 Microhardness evolution of the typical layers with (a) large grains and (b) small grains overall the rolling deformation at various nominal rolling strains

\section{Conclusion}

In conclusion, strain dependent grain growth behavior during cold rolling deformation of the electrodeposited grain-size gradient nanograined nickel was investigated. Two groups of gradient samples with similar component but different sequences were compared. Based on the microstructure observations and quantitative analysis, it can be found that there is a difference in growth rate between the layer with small grains $(\sim 20 \mathrm{~nm}$ determined by SEM) and the layer with large grains $(\sim 90 \mathrm{~nm}$ determined by SEM), resulting from strain gradient caused by inhomogeneous plastic deformation.

\section{Acknowledgement}

The authors acknowledge financial support from the National Natural Science Foundation of China (Grant No. 51601026) and Local Fund for Chongqing University of Arts and Sciences (Grant No. R2018SJD19).

\section{References}

1. Y.F. Wang, C.X. Huang, M.S. Wang, Y.S.Li, Y.T. Zhu. Quantifying the synergetic strengthening in gradient material. Scripta Mater., 2018, 150: 2225.

2. X.Y. Li, L. Lu, J.G. Li, X. Zhang, H.J. Gao.

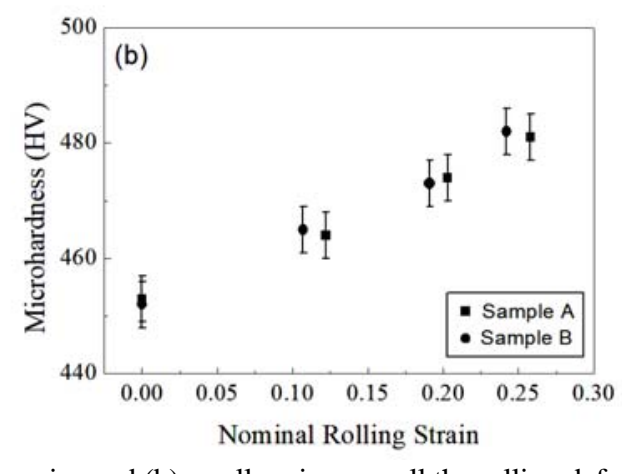

grains indeed lower than that for the layers with large grains. Therefore, as mentioned earlier, it is not difficult to understand the grain growth behavior in the present gradient design.

Mechanical properties and deformation mechanisms of gradient nanostructured metals and alloys. Nat. Rev. Mater., 2020, 5: 706-723.

3. J.J. Li, G.J. Weng, S.H. Chen, X.L. Wu. On strain hardening mechanism in gradient nanostructures. Int. J. Plasticity, 2017, 88: 89-107.

4. Y. Lin, J. Pan, H.F. Zhou, H.J. Gao, Y. Li. Mechanical properties and optimal grain size distribution profile of gradient grained nickel. Acta Mater., 2018, 153: 279-289.

5. Q.Y. Long, J.X. Lu, T.H.Fang. Microstructure and mechanical properties of AISI 316L steel with an inverse gradient nanostructure fabricated by electro-magnetic induction heating. Mater. Sci. Eng. A, 2019, 751: 42-50.

6. X.L. Wu, Y.Y. Zhu. Inverse Grain-Size Effect on Twinning in Nanocrystalline Ni. Phys. Rev. Lett., 2008, 101:025503.

7. H.T. Ni, X.Y. Zhang. Quantitative defect evolution of nanocrystalline nickel during cold rolling. Phil. Mag. Lett., 2012, 92:202-210.

8. H.T. Ni, L.X. Wang, Z.D. Wang, J. Zhu, Grain Orientation Induced Softening in Electrodeposited Gradient Nanostructured Nickel during Cold Rolling Deformation. Rev. Adv. Mater. Sci., 2020, 59:144-150

9. H.T. Ni, P. Li, Z.D. Wang, Z.X. Zou, M.C. Zhao, L.X. Wang, J. Zhu. Fabrication and 
Characterization of Nanocrystalline Nickel with a Grain Size Gradient by Direct Current Electrodeposition. Int. J. Electrochem. Sci., 2019, 14: 8429-8438.

10. W. Chen, Z.S. You, N.R. Tao, Z.H. Jin, L. Lu. Mechanically-induced grain coarsening in gradient nano-grained copper. Acta Materi., 2017, 125: 255-264.

11. K. Lu. Making strong nanomaterials ductile with gradients. Science, 2014, 345: 1455-1456. 